\title{
Statistical properties of superflares on solar-type stars based on 1-min cadence data
}

\author{
Hiroyuki Maehara ${ }^{*}$, Takuya Shibayama², Yuta Notsu³ ${ }^{3}$,Shota Notsu ${ }^{3}$, Satoshi Honda ${ }^{4}$, Daisaku Nogami ${ }^{3}$ \\ and Kazunari Shibata ${ }^{5}$
}

\begin{abstract}
We searched for superflares on solar-type stars using Kepler data with 1-min sampling in order to detect superflares with a short duration. We found 187 superflares on 23 solar-type stars whose bolometric energy ranges from the order of $10^{32}$ to $10^{36} \mathrm{erg}$. Some superflares show multiple peaks with the peak separation of the order of 100 to $1,000 \mathrm{~s}$ which is comparable to the periods of quasi-periodic pulsations in solar and stellar flares. Using these new data combined with the results from the data with 30-min sampling, we found that the occurrence frequency (dN/dE) of superflares as a function of flare energy (E) shows the power-law distribution ( $\mathrm{dN} / \mathrm{dE} \propto E^{-\alpha}$ ) with $\alpha \sim-1.5$ for $10^{33}<E<10^{36} \mathrm{erg}$ which is consistent with the previous results. The average occurrence rate of superflares with the energy of $10^{33} \mathrm{erg}$ which is equivalent to $X 100$ solar flares is about once in 500 to 600 years. The upper limit of energy released by superflares is basically comparable to a fraction of the magnetic energy stored near starspots which is estimated from the photometry.

We also found that the duration of superflares $(\tau)$ increases with the flare energy $(E)$ as $\tau \propto E^{0.39 \pm 0.03}$. This can be explained if we assume the time scale of flares is determined by the Alfvén time.
\end{abstract}

Keywords: Superflares; Solar flares; Stellar flares; Solar-type stars; Extreme space weather events

\section{Findings Introduction}

Solar flares are eruptive events in the solar atmosphere caused by the magnetic reconnection (e.g., Shibata and Magara 2011). The occurrence frequency of solar flares decreases as the flare energy increases. The frequencyenergy distribution of solar flares can be fitted by a simple power-law function with an index of -1.5 to -1.9 in the flare energy range between $10^{24}$ and $10^{32}$ erg (e.g., Aschwanden et al. 2000; Shimizu1995; Crosby et al. 1993). The total bolometric energy released by the largest solar flares is estimated to be the order of $10^{32} \mathrm{erg}$ (Emslie et al. 2012), and the occurrence frequency of such flare is about once in 10 years. On the other hand, much larger flares called 'superflares' have been observed on a wide variety of stars including solar-type stars (Landini et al. 1986; Schaefer 1989; Schaefer et al. 2000). Recently, many superflares on solar-type stars (G-type main sequence

\footnotetext{
*Correspondence: h.maehara@oao.nao.ac.jp

1 Okayama Astrophysical Observatory, National Astronomical Observatory of Japan, 3037-5 Honjo, Kamogata, Asakuchi, Okayama 719-0232, Japan

Full list of author information is available at the end of the article
}

stars) have been discovered by the Kepler space telescope (Maehara et al. 2012; Shibayama et al. 2013; Wu et al. 2015). The bolometric energy released by the superflare ranges from $10^{33}$ to $10^{36} \mathrm{erg}$ which is 10 to $10^{4}$ times larger than that of the largest solar flares $\left(10^{32} \mathrm{erg}\right)$. Although most of G-type main sequence stars exhibiting superflares are rapidly rotating stars, the temperature and rotation period of some superflare stars are close to those of the Sun. For example, (Nogami et al. 2014) reported that the rotational velocities of two superflare stars, KIC 9766237 and KIC 9944137, are comparable to that of our Sun, and no hint of binarity was detected. The average strength of the magnetic field on the surface of these stars, by using the absorption line of Ca II at $\lambda 8,542 \AA$, are estimated to be 1 to $20 \mathrm{G}$ which is comparable to or 1 order of magnitude higher than that of the Sun (approximately $2 \mathrm{G}$ ). On the other hand, (Wichmann et al. 2014) reported that some superflare stars with a photometric period of 10 to 20 days have large rotational velocities of $v \sin i \sim 10$ to $20 \mathrm{~km} / \mathrm{s}$. This suggests that these stars are either sub-giant stars or binary systems, not main sequence stars like our Sun.

\section{Springer}

(c) 2015 Maehara et al: licensee Springer. This is an Open Access article distributed under the terms of the Creative Commons Attribution License (http://creativecommons.org/licenses/by/4.0), which permits unrestricted use, distribution, and reproduction in any medium, provided the original work is properly credited. 
Solar flares are sometimes accompanied by coronal mass ejections (CMEs). Earth-directed CMEs can cause geomagnetic storms which can produce geomagnetically induced currents (GICs) in long electrical conductors (e.g., power lines) and can damage electrical equipment. For example, on 13 March 1989, about 6 million people in Quebec, Canada suffered an electrical power blackout caused by a huge geomagnetic storm (Allen et al. 1989). Moreover, in 1859, one of the largest solar flares, called the Carrington flare (Carrington 1859), caused the largest geomagnetic storm in the past 200 years (Tsurutani et al. 2003) which led to a failure of telegraph systems in Europe and North America (Loomis 1859). Even the solar flares which release up to $10^{32}$ erg of energy can damage not only our power grids but also communication and navigation systems. If superflares were to occur on the Sun, they would cause much more serious damage to our civilization. Therefore, it is important to study the properties of superflares on solar-type stars.

Previous studies of superflares on the basis of the Kepler data mainly focus on superflares with extremely large energy ( $>10^{34} \mathrm{erg}$ ) and long duration $(>1 \mathrm{~h})$ and could not discuss the statistical properties of superflares with energy between $10^{33}$ to $10^{34}$ erg due to low time resolution of the data. In this paper, we report the results from the data with high time resolution (approximately $1 \mathrm{~min}$ ) and discuss the statistical properties (e.g., occurrence frequency, flare duration) of superflares on solar-type stars.

\section{Methods and data}

We searched for flares (sudden brightenings of the star) from the short-cadence data (approximately 1-min interval) observed with the Kepler space telescope between April 2009 (quarter 0: Q0) and May 2013 (Q17) (Gilliland et al. 2010; Koch et al. 2010). We selected solar-type (G-type main sequence) stars from the data set by using the surface temperature of the star $\left(T_{\text {eff }}\right)$ and the surface gravity $(\log g)$ taken from Huber et al. (2014) instead of those from Brown et al. (2011) (initial Kepler Input Catalog). In previous papers (Maehara et al. 2012; Shibayama et al. 2013), we used stellar parameters taken from Brown et al. (2011) and the selection criteria of 5,100 K $<T_{\text {eff }}<$ $6,000 \mathrm{~K}$ and $\log g>4.0$. However, the temperatures in Brown et al. (2011) are systematically lower by approximately $200 \mathrm{~K}$ than those in Huber et al. (2014). Since the occurrence frequency of superflares depends on the surface temperature (e.g., Candelaresi et al. 2014), this systematic difference in surface temperature of stars would affect the resultant flare occurrence frequency. Therefore, in order to reduce the difference in the occurrence frequency of superflares caused by the systematic difference in temperature, we used the selection criteria of $5,300 \mathrm{~K}<$ $T_{\text {eff }}<6,300 \mathrm{~K}$ and $4.0<\log g<4.8$. The total number of solar-type stars observed with short-cadence mode is 1,547 .

We used the Presearch Data Conditioned (PDC) light curve (Stumpe et al. 2012; Smith et al. 2012) for the detection of flares. The typical length of each continuous observation in short-cadence mode is about 30 days. We first calculated the distribution of the following parameter $\left(\Delta F^{2}\right)$ for all consecutive data points from two pairs of the stellar brightness $(F(t))$ at the time of $t_{i-n-1}, t_{i-n}, t_{i}$, and $t_{i+1}\left(i\right.$ and $n$ are integer numbers and $t_{i}$ means time of the $i$ th data point):

$$
\Delta F^{2}\left(t_{i, n}\right)=s\left(F_{i}-F_{i-n-1}\right)\left(F_{i+1}-F_{i-n}\right),
$$

where

$$
s= \begin{cases}1 & \left(F_{i}-F_{i-n-1}>0 \text { and } F_{i+1}-F_{i-n}>0\right) \\ -1 & \left(F_{i}-F_{i-n-1}<0 \text { or } F_{i+1}-F_{i-n}<0\right)\end{cases}
$$

In the case of typical 30-day short-cadence data blocks, $i$ ranges from 1 to approximately 44,000. If the flare peak is around at $t_{i}$ and the stellar brightness at $t_{i-n}$ is close to the quiescent level, the value of $\Delta F^{2}\left(t_{i}\right)$ becomes much larger than the mode of the distribution. Therefore, $t_{i}-t_{i-n}$ should be comparable to or longer than the rise time of superflares. According to (Shibayama et al. 2013), the rise time of superflare on KIC 11610797 is about $2 \mathrm{~min}$. In the case of the X17 ${ }^{\mathrm{a}}$ solar flare (Kopp et al. 2005), the rise time is about $4 \mathrm{~min}$. Thus, we used the separation between two data points $n=3\left(t_{i}-t_{i-n} \sim 3 \mathrm{~min}\right.$ ) and $n=10$ (approximately $10 \mathrm{~min}$ ) for the analysis. In the case of larger $n$ value, we can detect flares having longer rise time.

The threshold of the flare was determined to be three times the value at the top $1 \%$ of the distribution.

We removed long-term brightness variations around the flare peak by fitting a quadratic function. We used the data from 0.05 to 0.01 (in the case of $n=3$ ) or from 0.15 to $0.03(n=10)$ days before the flare peak and the data from 0.05 to 0.10 day $(n=3)$ or 0.15 to 0.25 day $(n=10)$ after the peak for the fitting. After removing the long-term light variations, the flare start time and flare end time are defined to be the time at which the residuals of stellar brightness become larger and smaller than three times of the photometric error $\left(\sigma_{\text {photo }}\right.$ PDC flux errors given in the Kepler data), respectively. We analyzed only flares with the duration longer than $5 \mathrm{~min}$ (at least five consecutive data points exceed $3 \sigma_{\text {photo }}$ ) since it is difficult to distinguish between true flares and false events in the case of a single data point above the threshold (e.g., spikes in Figure 1h).

We excluded the flare candidates which have longer rise time than decay time. Since the number of stars observed in short-cadence mode is only approximately 1,500 , the possibility that two or more stars exhibit flares at the same time is negligible. Thus, we also removed the pairs of flare events on different stars which have the same flare peak 

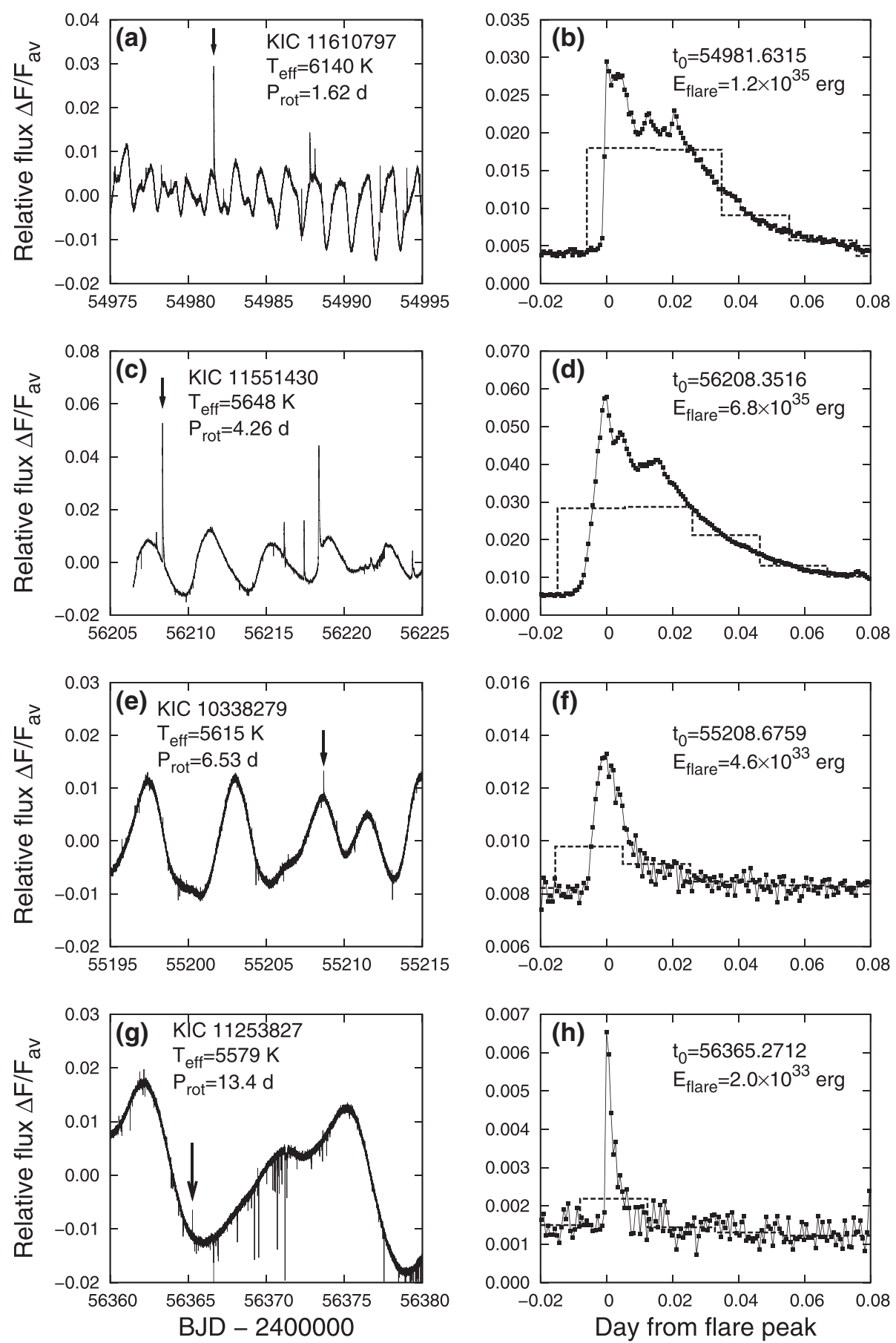

Figure 1 Light curves of typical superflares. (a) Long-term light variations of the G-dwarf KIC 11610797. The vertical axis means the relative difference between the observed brightness of the star and the average brightness during the observation period. The horizontal axis means the times of the observations in Barycentric Julian Date. (b) Enlarged light curve of a superflare observed around Barycentric Julian Date (BJD) 2454981.63 (indicated by the down arrow in panel (a)). Filled squares with solid lines and dashed lines represent the light curves from short- and long-cadence data, respectively. The long-cadence light curve is shifted in vertical direction for clarity. The flare peak ( $\left.t_{0}\right)$ is BJD 2454981.6315 and the bolometric energy of this flare $\left(E_{\text {flare }}\right)$ is $1.2 \times 10^{35} \mathrm{erg}$. (c) Same as panel (a), but for KIC 11551430. (d) Same as panel (b), but for the superflare observed on KIC 11551430 around BJD 2456208.35 (down arrow in panel (c)). (e) Same as panel (a), but for KIC 10338279. (f) Same as panel (b), but for the superflare observed on KIC 4543412 around BJD 2455208.68 (down arrow in panel (e)). (g) Same as panel (a), but for KIC 11253827. (h) Same as panel (b), but for the superflare observed on KIC 11253827 around BJD 2456365.27 (down arrow in panel (g)). 
time. After removing these false events, we checked all light curves of flare candidates by eye and removed nonflare events, since our flare detection method misidentified some eclipses or light curve jumps as flares. We also examined the pixel level data of flare stars in order to exclude the contamination of flares from nearby stars or other false events. If the spatial pixel distributions of the brightness of a star on CCDs are different between during the flare and at the quiescence, the candidate of a flare is revealed to be a light variation of nearby source or a non-astrophysical event (e.g., Maehara et al. 2012). We removed five events in this step.

We checked the Kepler Eclipsing Binary Catalog (Kirk 2013; Prša A et al. 2011; Slawson et al. 2011) and removed all flare candidates which occurred on the eclipsing binary (14 stars). We also removed all flare candidates on the stars which are listed as visual or spectroscopic binaries in Notsu et al. (2015a) (2 stars). In total, we eliminated 152 flare candidates on these 16 stars. Since the fraction of known binary systems would be small, there may be more unknown binary systems in the sample. According to Notsu et al. (2015a), the lower limit of binary fraction among superflare stars based on high-dispersion spectroscopy is about $30 \%$, which is not so different than the binary fraction of nearby solar-type stars (e.g., Duquennoy and Mayor 1991).

Bolometric energy emitted by each flare was estimated from the stellar luminosity, flare amplitude, and flare duration with the same manner as Shibayama et al. (2013). The luminosity of each flare star were estimated from the effective temperature and the stellar radius taken from (Huber et al. 2014). The error in bolometric energy was estimated from errors in the temperature and stellar radius from (Huber et al. 2014). Since the error in the temperature is $\sigma_{T_{\text {star }}} \sim 200 \mathrm{~K}\left(\sigma_{T_{\text {star }}} / T_{\text {star }} \sim 3 \%\right.$ to $\left.4 \%\right)$ and that in the stellar radius is $\sigma_{R_{\mathrm{star}}} / R_{\mathrm{star}} \sim 10 \%$, the typical error in bolometric energy would be $\sigma_{E_{\text {flare }}} / E_{\text {flare }} \sim 25 \%$.

We estimated periods of long-term light variations from light curves of each star by using the discrete Fourier transform (DFT) method. For period analysis, we used the long-cadence data (time resolution of $30 \mathrm{~min}$ ) between September 2009 (Q2) and April 2013 (Q16). After removing linear trends in each quarter and correcting the difference in mean flux between different quarters, we calculated the power spectrum and chose a peak corresponding to the maximum power among peaks which are 20 times larger than the noise level.

\section{Results and discussion}

We detected 187 flares on 23 solar-type stars from the data of 1,547 solar-type stars. The details of all detected flares and properties of superflare stars are summarized in Additional file 1: Tables S1 to S6 (CSV version of Table S1 and Tables $\mathrm{S} 2$ to $\mathrm{S} 6$ are also available as Additional files 2 and 3, respectively). Figure 1 shows typical light curves of superflares from short-cadence data. Light curves of all detected flares are shown in Additional file 1: Figures S1 to S94. The amplitudes of flares in a unit of average brightness of the star range from $1.3 \times 10^{-3}$ to $8.5 \times 10^{-2}$, and the bolometric energies of flares range from $2 \times 10^{32}$ to $8 \times 10^{35} \mathrm{erg}$. The lower end of the amplitude of detected flare is comparable to that of the X17 class solar flare (normalized amplitude $\sim 3 \times 10^{-4}$ ) occurred on 28 October 2003 (Kopp et al. 2005). As shown in Figure 1b,d, some flares show multiple peaks with the peak separation of 100 to $1,000 \mathrm{~s}$. The time scale of these time variations is comparable to that of flare oscillations observed in the Sun (e.g., Aschwanden et al. 1999; Nakariakov and Melnikov 2009) and M dwarfs (Anfinogentov et al. 2013; MitraKraev et al. 2005; Welsh et al. 2006). On the analogy of the solar flare oscillations, these multiple peaks may be caused by coronal loop oscillations associated with flares.

\section{Occurrence frequency distribution}

Figure 2 represents the occurrence frequency distribution of superflares with energy $>10^{33} \mathrm{erg}$ as a function of the rotation period. The flare frequency strongly depends on the rotation period, and the frequency rapidly decreases as the rotation period increases in the period above a few days. No flare was detected on the stars with $P_{\text {rot }} \geq 20$ days from short-cadence data, and the upper limit of the frequency for this bin is $<1$ in 80 years. The flare occurrence frequency on the stars with $2.5 \leq P_{\text {rot }}<20$ days is roughly proportional to $P_{\text {rot }}^{-3}$. The flare frequency on the stars with $P_{\text {rot }} \geq 20$ days expected from this decrease trend is approximately 0.004 year $^{-1} \mathrm{star}^{-1}$ which is about eight times lower than that on the stars with $10 \leq P_{\text {rot }}<$ 20 days.

The similar relation between flare frequency and rotation period was reported by previous studies using the long-cadence data (e.g., Notsu et al. 2013b).

The rotation period is thought to be related to the age of stars (e.g., Ayres 1997). This result suggests that the young solar-type stars would exhibit superflares more frequently.

Figure 3a represents the occurrence frequency of superflares as a function of the bolometric energy of superflares. The solid histogram in Figure $3 a$ represents the frequency distribution of superflares on all solartype stars derived from short-cadence data, and the dashed histogram represents that from long-cadence data (Shibayama et al. 2013).

The average flare frequencies in a given energy bin from short-cadence data are about five times higher than those from long-cadence data. This difference in the flare frequency is mainly caused by that in the rotation period distribution of the observed targets. The period distribution of the targets in short-cadence mode is biased to the shorter-period end. The fraction of stars with the 


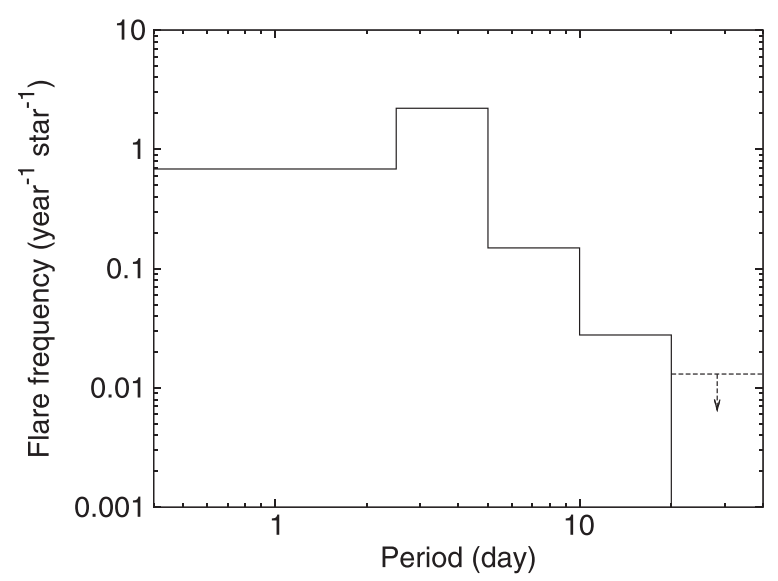

Figure 2 Occurrence frequency distribution of superflares as a function of the rotation period. The horizontal axis indicates the rotation period of the star derived from photometric data. The vertical axis indicates the number of superflares per star and per year in each period bin. Period bins are defined as follows: $P<2.5$ days, $2.5 \leq P<5$ days, $5 \leq P<10$ days, $10 \leq P<20$ days, and $P \geq 20$ days. Solid line histogram represents the occurrence frequency of superflares with an energy of $>10^{33} \mathrm{erg}$ in each period bin. The dashed down arrow indicates the upper limit of the flare frequency.

rotation period $\left(P_{\text {rot }}\right)<10$ days observed in long-cadence mode is $8.1 \%(8312 / 102709)$. On the other hand, that of stars with $P_{\text {rot }}<10$ days in short-cadence mode is $32 \%(499 / 1547)$. As mentioned above, the stars with shorter rotation period exhibit more frequent superflares. Therefore, the average flare frequency derived from shortcadence data is higher than that from long-cadence data.

The solid histogram in Figure $3 \mathrm{~b}$ represents the corrected frequency distribution of superflares from shortcadence data taking into account the difference in the period distribution of the observed samples. Since the ratio of the total observation time (sum of the length of observation time for each star) for stars with $P_{\text {rot }}<$ 10 days to that for stars with $P_{\text {rot }} \geq 10$ days in shortcadence mode (1:1.7) is about 6.5 times larger than the ratio in long-cadence mode $(1: 11)$, we calculated the corrected occurrence frequency by adjusting the ratio of total observation time in short-cadence mode to that in longcadence mode. Both frequency distributions from shortand long-cadence data are almost the same for the flare energy between $10^{34}$ and $10^{36} \mathrm{erg}$. The frequency of flares derived from the long-cadence data is less than that from the short-cadence data for the flare energy below $10^{34} \mathrm{erg}$. This difference is caused by the detection limit of flare search method. Since the time resolution of long-cadence data is much lower than that of short-cadence data, we could not detect smaller flares from long-cadence data, and the observed flare frequency from long-cadence data becomes much lower than that from short-cadence data.

The frequency distribution of superflares from the short-cadence data can be fitted by a power-law function with the index of $-1.4 \pm 0.2$ for the energy between $4 \times 10^{33}$ and $1 \times 10^{36} \mathrm{erg}$. This value is comparable to the power-law index for the frequency distribution of superflares derived from the long-cadence data. Using the combined data set from both short- and long-cadence data, the power-law index is $-1.5 \pm 0.1$ for the flare energy of $4 \times 10^{33}$ to $1 \times 10^{36} \mathrm{erg}$.

(Shibata et al. 2013) pointed out that the frequency distribution of superflares on Sun-like stars (early-G dwarfs with $P_{\text {rot }} \geq 10$ days) and those of solar flares are roughly on the same power-law line. Figure 4 represents the comparison between the frequency distribution of superflares on Sun-like stars $\left(5,800 \leq T_{\text {eff }}<6,300\right.$ and $P_{\text {rot }} \geq 10$ days) derived from both short- (filled circles) and longcadence (solid line) data and those of solar flares (dashed lines). The thin dotted line indicates the power-law function with an index of -1.8 taken from Shibata et al. (2013). The frequency distribution of superflares derived from short-cadence data is also on the same power-law line. This distribution suggests that the average occurrence frequency of superflares on Sun-like stars with bolometric energy of $10^{33}, 10^{34}$, and $10^{35}$ erg are once in approximately 70 years, approximately 500 years, and approximately 4,000 years, respectively. As mentioned in the previous paragraph, the frequency of superflares strongly depends on the rotation period. The flare frequency on the stars with the same rotation period as the Sun $\left(P_{\text {rot }} \sim\right.$ 25 days) would be approximately eight times smaller that that on the stars with $10 \leq P_{\text {rot }}<20$ days. Therefore, the occurrence frequency of superflares with energy $>10^{33}$ erg would be once in 500 to 600 years. These values are lower than the upper limits derived from radionuclides in lunar samples (Schrijver et al. 2012). Miyake et al. (2012) reported a rapid increase in the ${ }^{14} \mathrm{C}$ content of tree rings around A.D. 774 to 775, and Miyake et al. (2013) found 

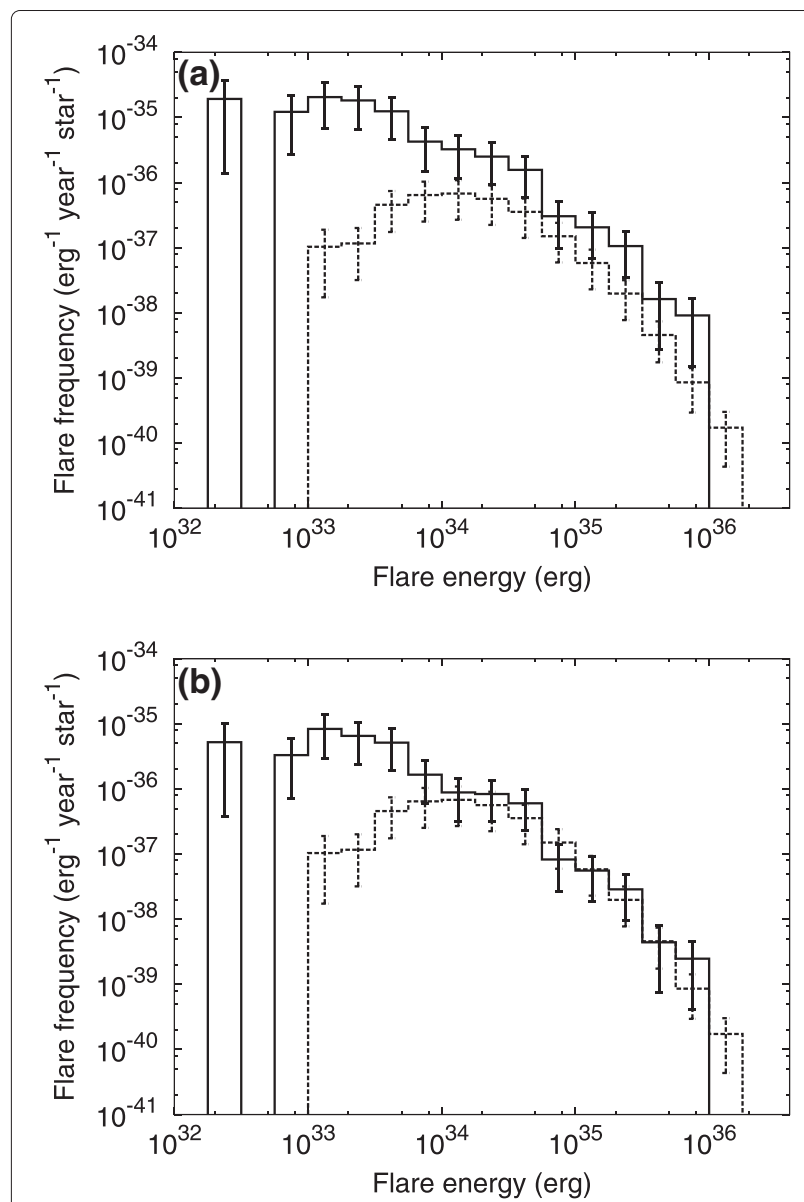

Figure 3 Occurrence frequency distribution of superflares as a function of flare energy. (a) Bold solid and dashed histograms represent the occurrence frequency of superflares on all G-type main sequence stars detected from short- (this work) and long-cadence data (Shibayama et al. 2013) as a function of total energy of superflares, respectively. The vertical axis indicates the number of superflares per star, per year, and per unit energy. Error bars represent $1-\sigma$ uncertainty in the frequency estimated from the square root of the number of detected flares in each energy bin and the uncertainty in flare energy. (b) Same as (a), but for the corrected frequency distribution from the short-cadence data which was calculated so that the ratio of total observation time in short-cadence mode is the same as that in long-cadence mode (see text for details of the correction).

another ${ }^{14} \mathrm{C}$ increase event around A.D. 993 to 994 . The cosmic ray flux of these events are equivalent to solar flares with the energy of approximately $10^{34} \mathrm{ergs}$, if cosmic ray sources of these events were solar flares. The occurrence frequency of these events (two events in 270 years) is roughly comparable to the average occurrence frequency of superflares on Sun-like stars with the energy of $10^{33}$ to $10^{34} \mathrm{erg}$. In the case of solar flares, the fraction of geo-effective CMEs is less than 10\% (e.g., Gopalswamy et al. 2007). If the fraction is the same for superflares on our Sun, the occurrence rate of extreme GIC events (ten

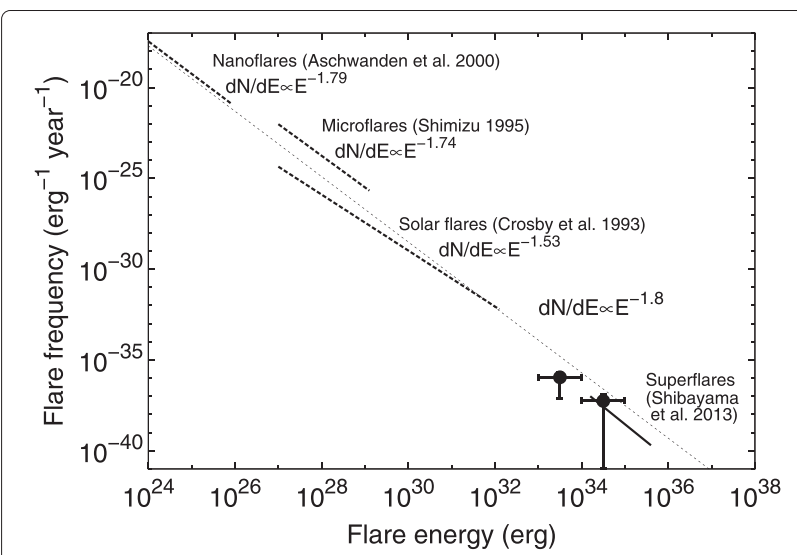

Figure 4 The comparison between the frequency distribution of superflares and solar flares. Filled circles indicate the occurrence frequency distribution of superflares on Sun-like stars (G-type main sequence stars with $P_{\text {rot }}>10$ days and 5,800 $<T_{\text {eff }}<6,300 \mathrm{~K}$ ) derived from short-cadence data. Horizontal error bars represent the range of each energy bin. The definition of the vertical error bars is the same as Figure 3. Bold solid line represents the power-law frequency distribution of superflares on Sun-like stars taken from (Shibayama et al. 2013). Dashed lines indicate the power-law frequency distribution of solar flares observed in hard X-ray (Crosby et al. 1993), soft X-ray (Shimizu 1995), and EUV (Aschwanden et al. 2000). Occurrence frequency distributions of superflares on Sun-like stars and solar flares are roughly on the same power-law line with an index of -1.8 (thin solid line) for the wide energy range between $10^{24}$ and $10^{35} \mathrm{erg}$.

times or more larger than the Carrington event) would be less than once in a few thousand years.

\section{Relation between flare energy and area of starspots}

Most of superflare stars show large-amplitude light variations with periods from a few days to approximately 20 days. If we assume these quasi-periodic variations are caused by the rotation of the star, the amplitude and period of light variations correspond to the area of starspots and the rotation period (Notsu et al. 2013b). The large-amplitude light variations of superflare stars suggest that the surface of superflare stars is covered by large starspots.

Figure 5 shows the scatter plot of flare energy as a function of the area of the starspot group $\left(A_{\mathrm{spot}}\right)$. The area of starspots on superflare stars were estimated from the amplitude of light variations $\left(\Delta F_{\text {rot }}\right)$ by using the following equation:

$$
A_{\text {spot }}=\Delta F_{\text {rot }} A_{\text {star }}\left\{1-\left(\frac{T_{\text {spot }}}{T_{\text {star }}}\right)^{4}\right\}^{-1},
$$

where $A_{\text {star }}$ is the apparent area of the star and $T_{\text {spot }}$ and $T_{\text {star }}$ are the temperatures of the starspot and photosphere of the star (Notsu et al. 2013b), respectively. Here, we define the amplitude as the brightness range normalized by the average stellar brightness, in which the lower $99 \%$ 


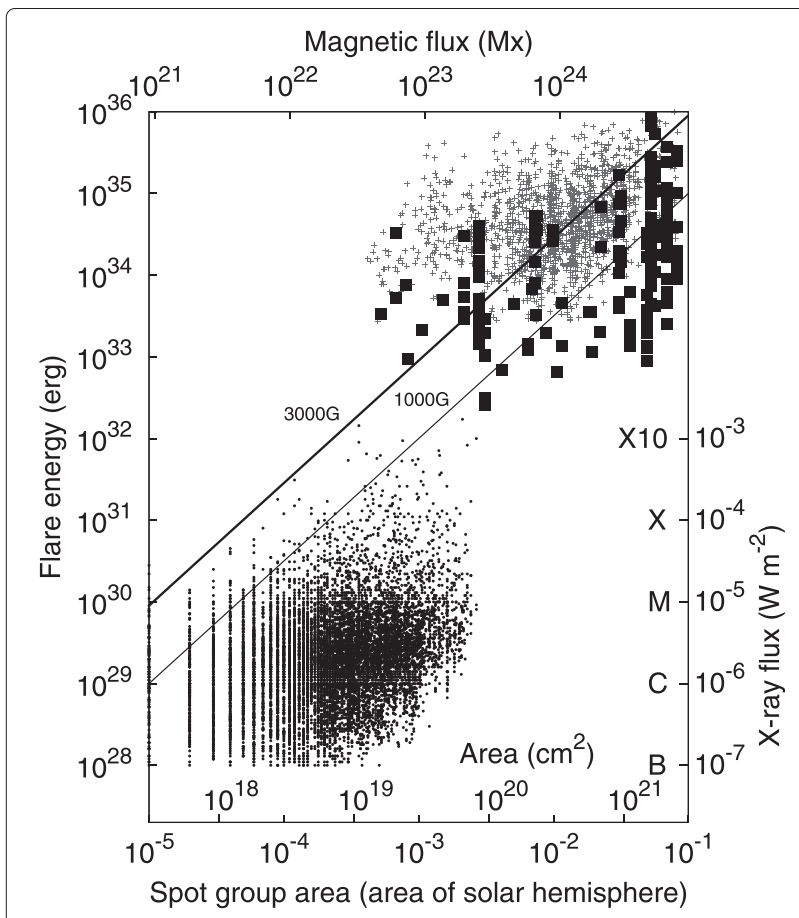

Figure 5 Scatter plot of flare energy as a function of spot area. The lower and upper horizontal axes indicate the area of the starspot group in the unit of the area of the solar hemisphere and the magnetic flux for $B=3,000 \mathrm{G}$. The vertical axis represents the bolometric energy released by each flare. Filled squares and small crosses indicate superflares on G-type main sequence stars detected from short- (this work) and long-cadence data (Shibayama et al. 2013), respectively. Small filled circles represent solar flares (Ishii et al., private communication, based on the data retrieved from the website of the National Geophysical Data Center of the National Oceanic and Atmospheric Administration (NOAA/NGDC), Solar-Terrestrial Physics Division at http://www.ngdc.noaa.gov/stp/). The area of the starspot group for G-type main sequence stars was estimated from the full amplitude of light variations during each observation period in which the flare occurred using Equation 3. We assumed that bolometric energies of $\mathrm{B}, \mathrm{C}, \mathrm{M}, \mathrm{X}$, and $\mathrm{X} 10$ class solar flares are $10^{28}, 10^{29}, 10^{30}$, $10^{31}$, and $10^{32}$ erg from observational estimates of energies of typical solar flares (e.g., Benz 2008; Emslie et al. 2012).

of the distribution of the brightness difference from average, except for the flares, are included. We estimated the amplitude from the long-cadence data of the quarter at which each superflares detected from the short-cadence data occurred. $A_{\text {star }}$ and $T_{\text {star }}$ for each superflare star were taken from Huber et al. (2014), and we assumed $T_{\text {spot }}=$ $4,000 \mathrm{~K}$ (Berdyugina 2005). Although there is a large scatter, the majority of energetic flares occur on the stars with large starspots. Since the flares are a result of a sudden conversion of magnetic energy to other forms (i.e., thermal and kinetic energy, e.g., Shibata and Magara (2011)), the flare energy is supplied from the magnetic energy stored near the starspots $\left(E_{\mathrm{mag}}\right)$ and the bolometric energy released by the flare may be a fraction of the magnetic energy (Maehara et al.2012; Notsu et al. 2013b; Shibata et al. 2013).

Here, the stored magnetic energy can be roughly estimated by:

$$
E_{\mathrm{mag}} \sim \frac{B^{2} L^{3}}{8 \pi}
$$

where $B$ and $L$ correspond to the magnetic field strength of the starspots and the scale length of the starspot group, respectively. By using the total area of the starspot group $A_{\text {spot }} \sim L^{2}$, the energy released by a flare can be written as:

$$
E_{\text {flare }} \sim f E_{\text {mag }} \sim \frac{f B^{2} A_{\text {spot }}^{3 / 2}}{8 \pi},
$$

where $f$ is the fraction of magnetic energy released by the flare. This suggests that the flare energy is roughly proportional to the area of the starspot group to the power of $3 / 2$. The typical value of $f$ is in the order of 0.1 (Aschwanden et al. 2014), the typical sunspot area for generating X10 class flares (approximately $10^{32} \mathrm{erg}$ ) observed in 1989 to 1997 was $3 \times 10^{-4}$ of the half area of the solar surface (Sammis et al. 2000), and the typical magnetic field strength of the sunspot is in the order of 1,000 G (e.g., Antia et al. 2003). Hence, equation 5 can be written as:

$E_{\text {flare }} \sim 7 \times 10^{32}(\mathrm{erg})\left(\frac{f}{0.1}\right)\left(\frac{B}{1,000 \mathrm{G}}\right)^{2}\left[\frac{A_{\text {spot }} / 2 \pi R_{\odot}^{2}}{0.001}\right]^{3 / 2}$.

Solid and dashed lines in Figure 5 represent Equation 6 for $f=0.1, B=1,000$ and 3,000 G, respectively. Majority of superflares detected from short-cadence data (filled squares) and almost all solar flares (small dots) are below these lines. This suggests that the upper limit of the energy released by the flare is basically determined by the magnetic energy estimated from the area of starspots. However, the bolometric energies of superflares detected from long-cadence data (small crosses) are biased to largerenergy end. As mentioned above, due to the low time resolution, only superflares with larger bolometric energies could be detected from long-cadence data. In addition, superflares with large energy but small occurrence frequency could be detected from the long-cadence data because of the large number of stars (approximately $10^{5}$ ).

There are some superflares above the analytic relation. KIC 7093428 is one of superflare stars exhibiting superflares whose energy is much larger than that expected from Equation 6. This object is located within the error circle of an X-ray source 1SXPS J184925.7+423901 (Evans et al. 2014). The X-ray luminosity of the object is approximately $10^{28} \mathrm{erg} / \mathrm{s}$ if the distance of the object is approximately 200 pc. The empirical relation between the X-ray luminosity and the magnetic flux of the star (Pevtsov et al. 2003) suggests that the magnetic flux of KIC 7093428 
is in the order of $10^{24} \mathrm{Mx}$. This value corresponds to the spot area of $3 \times 10^{20} \mathrm{~cm}^{2}$ for $B=3,000 \mathrm{G}$, and the energy of the largest superflare on KIC 7093428 is comparable to the flare energy estimated from Equation 6. The spot area of the object expected from the X-ray luminosity is more than 1 order of magnitude larger than that estimated from the amplitude of quasi-periodic modulations. The amplitude of light variations due to the rotation of star is affected by the inclination of rotation axis and the latitude of starspots (e.g., Notsu et al. 2013a,b). In the case of stars with low-inclination angle or stars with starspots around the polar region, the light variation caused by the rotation becomes small, and the area of starspots derived from the amplitude of light variation is smaller than the actual area. According to Notsu et al. (2015b), some superflare stars which show superflares with energy larger than that expected from the amplitude of light variation have low-inclination angles.

In addition, polar spots are often observed on rapidly rotating cool stars (e.g., Strassmeier 2009). Another possibility is flares on the companion star. (Kitze et al. 2014) found that the astrometric position of the Sun-like superflare star, KIC 7133671 which shows a superflare with energy larger than expected from the photometric spot area, was shifted by 25 mas during the flare. This suggests that the flare on KIC 7133671 occurred on a faint companion star. In this case, the flare energy would not be related to the area of the starspot group on the primary star.

\section{Correlation between flare duration and flare energy}

Figure 6 shows the duration of superflares as a function of the bolometric energy of flares. Here, we defined the flare duration as the $e$-folding decay time of flare intensity after its peak. The duration of superflares increases as the flare energy increases. The linear fit for the superflares from short-cadence data in the log-log plot yields:

$$
\tau_{\text {flare }} \propto E_{\text {flare }}^{0.39 \pm 0.03}
$$

where $\tau_{\text {flare }}$ and $E_{\text {flare }}$ indicate the duration and bolometric energy of flares. The duration of superflares derived from long-cadence data (crosses) is longer than that from short-cadence data. This difference may be caused by the difference in time resolution of the data and selection bias. Since the time resolution of long-cadence data is $30 \mathrm{~min}$ and much longer than that of short-cadence data $(1 \mathrm{~min})$, the flare detection method for long-cadence data used in Maehara et al. (2012) and Shibayama et al. (2013) can detect only superflares with long duration. The bolometric energy and duration in optical wavelength of the X17 solar flare on 28 October 2003 are in the order of $10^{32}$ erg and approximately $10 \mathrm{~min}$ (Kopp et al. 2005), which are roughly comparable to the correlation between the duration and energy of superflares on solar-type stars.

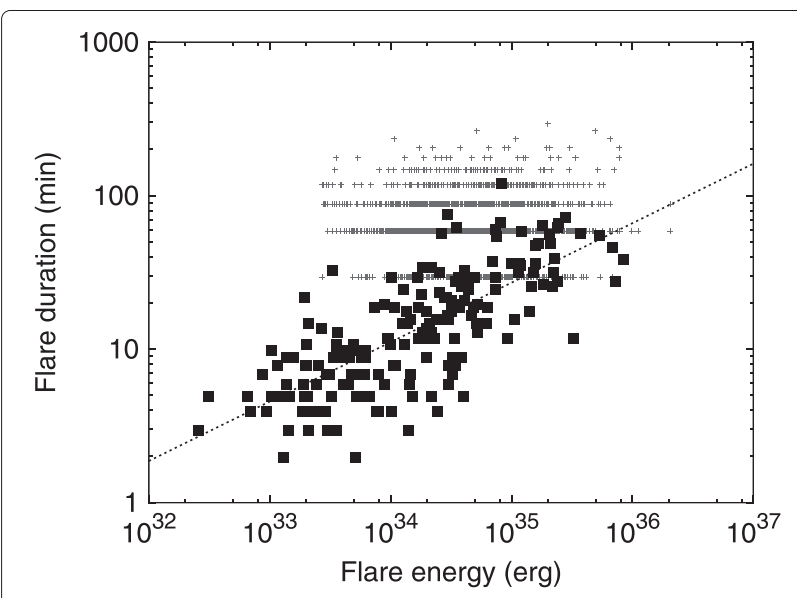

Figure 6 Scatter plot of the duration of superflares as a function of the bolometric energy. Filled squares and small crosses indicate superflares on G-type main sequence stars detected from short- and long-cadence data, respectively. We used e-folding decay time as the flare duration. Dotted line indicates the linear regression for the data of superflares from short-cadence data. The power-law slope of the line is $0.39 \pm 0.03$.

Similar correlation between the flare duration and energy was observed in solar flares. The power-law slope for the correlation between the duration of solar flares and X-ray fluence observed with the GOES is about $1 / 3$ (Veronig et al. 2002). The correlation between the duration and peak flux observed with RESSI also shows similar power-law slope of approximately 0.2 (Christe et al. 2008). These similarity between solar flares and superflares on solar-type stars suggests that solar flares and superflares are caused by the same physical process (i.e., reconnection).

As discussed in the previous subsection, the flare energy is related to the magnetic energy stored near the starspots as follows:

$$
E_{\text {flare }} \sim f E_{\text {mag }} \propto f B^{2} L^{3},
$$

where $f, B$, and $L$ correspond to the fraction of energy released by a flare, the magnetic field strength of the starspots and the scale length of the starspot group respectively. On the other hand, the duration of flares, especially the duration of impulsive phase of flares, is comparable to the reconnection time $\left(\tau_{\text {rec }}\right)$ which can be written as

$$
\tau_{\text {flare }} \sim \tau_{\text {rec }} \sim \tau_{\mathrm{A}} / M_{\mathrm{A}} \sim L / v_{\mathrm{A}} / M_{\mathrm{A}},
$$

where $\tau_{\mathrm{A}}=L / v_{\mathrm{A}}$ is the Alfvén time, $v_{\mathrm{A}}$ is the Alfvén velocity, and $M_{\mathrm{A}}$ is the non-dimensional reconnection rate which is comparable to 0.1 to 0.01 for the fast reconnection such as the Petschek model (e.g., Shibata and Magara 2011). Since all G-type main sequence stars have similar stellar properties (e.g., $T_{\text {eff }}$ and $\log g$ ), $B$ and $v_{\mathrm{A}}$ 
might not be so different among them. Therefore, from Equations 8 and 9, the duration of flares can be written as:

$$
\tau_{\text {flare }} \propto E_{\text {flare }}^{1 / 3} .
$$

This suggests that the power-law slope for the correlation between the flare duration and flare energy is about $1 / 3$, and this is comparable to the observed value of $0.39 \pm 0.03$.

\section{Conclusions}

We found 187 superflares on 23 solar-type stars from the 1-min cadence data obtained with the Kepler space telescope. Because of the high temporal resolution of the data, we found the following new results:

1. The power-law frequency distribution of superflares (e.g., Maehara et al. 2012; Shibayama et al. 2013) continues to the flare energy of $10^{33} \mathrm{erg}$, and the average occurrence rate of the X100 superflares on the stars with similar rotation period to the Sun is about once in 500 to 600 years.

2. Some of the superflares show multiple peaks during a flare with the peak separation of 100 to $1,000 \mathrm{~s}$. The time scale of these modulation during the flare is comparable to the periods of quasi-periodic pulsations in solar and stellar flares.

3. The flare duration increases with the flare energy as $\tau_{\text {flare }} \propto E_{\text {flare }}^{0.39 \pm 0.03}$

Observations of solar flares, especially white light flares, with the high temporal resolution are also important to compare the properties of solar flares and those of superflares on solar-type stars.

\section{Endnote}

${ }^{a}$ Solar flares are classified as A, B, C, M, or X, according to the peak X-ray ( 1 to $8 \AA$ ) flux as measured by the GOES satellite. Peak fluxes of A, B, C, M, and X class solar flares are $<10^{-7}, 10^{-7}$ to $10^{-6}, 10^{-6}$ to $10^{-5}$, $10^{-5}$ to $10^{-4}$, and $\geq 10^{-4} \mathrm{~W} / \mathrm{m}$, respectively. Each $\mathrm{X}$-ray class is divided into a linear scale from 1 to 9 (e.g., B1 to B9, $\mathrm{C} 1$ to C9, and M1 to M9). An X17 class flare is 17 times more energetic than an X1 class flare.

\section{Additional files}

\footnotetext{
Additional file 1: Supplementary tables and figures. This file contains a table summarizing the stellar parameters of superflare stars (Table S1), list of detected superflares (Tables S2 to S6), and light curves of detected superflares (Figures S1 to S94).

Additional file 2: Stellar parameters of superflare stars. This file is the version of Table $\mathbf{S} \mathbf{1}$ in Additional file 1.

Additional file 3: List of superflares. This file is the version of Tables S2 to $\mathbf{S 5}$ in Additional file 1.
}

\section{Competing interests}

The authors declare that they have no competing interests.

\section{Authors' contributions}

HM developed the flare detection method and was responsible for the data analysis. He also wrote the majority of the text. TS contributed to the analysis of stellar flares observed by the Kepler. SN and YN analyzed the rotation of stars observed by the Kepler. SH and DN contributed to the interpretation of the stellar brightness variation and helped in the data analysis from the general stellar astronomical point of view. KS provided theoretical interpretation of the observations and provided advice on the paper's content. All authors read and approved the final manuscript.

\section{Acknowledgements}

Kepler was selected as the tenth Discovery mission. The funding for this mission is provided by the NASA Science Mission Directorate. The data presented in this paper were obtained from the Multimission Archive at STSCl. This work was supported by MEXT/JSPS KAKENHI Grant Numbers 25287039, 26400231, and 26800096.

\section{Author details}

${ }^{1}$ Okayama Astrophysical Observatory, National Astronomical Observatory of Japan, 3037-5 Honjo, Kamogata, Asakuchi, Okayama 719-0232, Japan.

${ }^{2}$ Solar-Terrestrial Environment Laboratory, Nagoya University, Furo-cho,

Chikusa-ku, Nagoya, Aichi 464-8601, Japan. ${ }^{3}$ Department of Astronomy, Kyoto University, Kitashirakawa-Oiwake-cho, Sakyo-ku, Kyoto, Kyoto 606-8502, Japan. ${ }^{4}$ Center for Astronomy, University of Hyogo, 407-2 Nishigaichi, Sayo-cho, Sayo, Hyogo 679-5313, Japan. ${ }^{5}$ Kwasan and Hida Observatories, Kyoto University, Yamashina-ku, Kyoto, Kyoto 607-8471, Japan.

Received: 30 September 2014 Accepted: 23 March 2015

Published online: 29 April 2015

\section{References}

Allen J, Sauer H, Frank L, Reiff P (1989) Effects of the March 1989 solar activity. Eos, Trans Am Geophys Union 70:1479-1488. doi:10.1029/89E000409

Anfinogentov S, Nakariakov VM, Mathioudakis M, Van Doorsselaere T, Kowalski AF (2013) The decaying long-period oscillation of a stellar megaflare. Astrophysical J 773:156. doi:10.1088/0004-637X/773/2/156

Antia HM, Bhatnagar A, Ulmschneider P (2003) Lectures on solar physics. Springer Verlag, Berlin

Aschwanden MJ, Fletcher L, Schrijver CJ, Alexander D (1999) Coronal loop oscillations observed with the transition region and coronal explorer. Astrophysical J 520:880-894. doi:10.1086/307502

Aschwanden MJ, Tarbell TD, Nightingale RW, Schrijver CJ, Title A, Kankelborg CC, Martens P, Warren HP (2000) Time variability of the "quiet" sun observed with TRACE. II. Physical parameters, temperature evolution, and energetics of extreme-ultraviolet nanoflares. Astrophysical J 535:1047-1065. doi:10.1086/308867

Aschwanden MJ, Xu Y, Jing J (2014) Global energetics of solar flares. I. Magnetic energies. Astrophysical J 797:50. doi:10.1088/0004-637X/797/1/50

Ayres TR (1997) Evolution of the solar ionizing flux. J Geophys Res 102:1641-1652. doi:10.1029/96JE03306

Benz AO (2008) Flare observations. Living Rev Solar Phys 5:1 doi:10.12942/Irsp-2008-1

Berdyugina SV (2005) Starspots: a key to the stellar dynamo. Living Rev Solar Phys 2:8. doi:10.12942/Irsp-2005-8

Brown TM, Latham DW, Everett ME, Esquerdo GA (2011) Kepler input catalog: photometric calibration and stellar classification. Astrophysical J 142:112. doi:10.1088/0004-6256/142/4/112

Candelaresi S, Hillier A, Maehara H, Brandenburg A, Shibata K (2014) Superflare occurrence and energies on G, K and M type dwarfs. Astrophysical J 792:67. doi:10.1088/0004-637X/792/1/67

Carrington RC (1859) Description of a singular appearance seen in the Sun on September 1, 1859. Mon Notices R Astronomical Soc 20:13-15

Christe S, Hannah IG, Krucker S, McTiernan J, Lin RP (2008) RHESSI microflare statistics. I. Flare-finding and frequency distributions. Astrophysical J 677:1385-1394. doi:10.1086/529011

Crosby NB, Aschwanden MJ, Dennis BR (1993) Frequency distributions and correlations of solar X-ray flare parameters. Solar Phys 143:275-299. doi:10.1007/BF00646488

Duquennoy A, Mayor M (1991) Multiplicity among solar-type stars in the solar neighbourhood. II - Distribution of the orbital elements in an unbiased sample. Astronomy Astrophysics 248:485-524 
Emslie AG, Dennis BR, Shih AY, Chamberlin PC, Mewaldt RA, Moore CS, Share GH, Vourlidas A, Welsch BT (2012) Global energetics of thirty-eight large solar eruptive events. Astrophysical J 759:71. doi:10.1088/0004-637X/759/1/71

Evans PA, Osborne JP, Beardmore AP, Page KL, Willingale R, Mountford CJ, Pagani C, Burrows DN, Kennea JA, Perri M, Tagliaferri G, Gehrels N (2014) 1SXPS: a deep swift X-ray telescope point source catalog with light curves and spectra. Astrophysical J Suppl 210:8. doi:10.1088/0067-0049/210/1/8

Gilliland RL, Jenkins JM, Borucki WJ, Bryson ST, Caldwell DA, Clarke BD, Dotson UL, Haas MR, Hall J, Klaus T, Koch D, McCauliff S, Quintana EV, Twicken JD, van Cleve JE (2010) Initial characteristics of Kepler short cadence data. Astrophysical J Lett 713:L160-L163. doi:10.1088/2041-8205/713/2/L160

Gopalswamy N, Yashiro S, Akiyama S (2007) Geoeffectiveness of halo coronal mass ejections. J Geophys Res (Space Physics) 112:6112. doi:10.1029/2006JA012149

Huber D, Silva Aguirre V, Matthews JM, Pinsonneault MH, Gaidos E, García RA, Hekker S, Mathur S, Mosser B, Torres G, Bastien FA, Basu S, Bedding TR, Chaplin WJ, Demory B-O, Fleming SW, Guo Z, Mann AW, Rowe JF, Serenelli AM, Smith MA, Stello D (2014) Revised stellar properties of Kepler targets for the quarter 1-16 transit detection run. Astrophysical J Suppl 211: article id:2. doi:10.1088/0067-0049/211/1/2

Koch DG, Borucki WJ, Basri G, Batalha NM, Brown TM, Caldwell D, Christensen-Dalsgaard J, Cochran WD, DeVore E, Dunham EW, Gautier TNIII, Geary JC, Gilliland RL, Gould A, Jenkins J, Kondo Y, Latham DW, Lissauer JJ, Marcy G, Monet D, Sasselov D, Boss A, Brownlee D, Caldwell J, Dupree AK, Howell SB, Kjeldsen H, Meibom S, Morrison D, Owen T, et al (2010) Kepler mission design, realized photometric performance, and early science. Astrophysical J Lett 713:L79-L86. doi:10.1088/2041-8205/713/2/L79

Kopp G, Lawrence G, Rottman G (2005) The Total Irradiance Monitor (TIM): science results. Solar Phys 230:129-139. doi:10.1007/s11207-005-7433-9

Kirk etal (2013) Kepler eclipsing binary catalog - third revision (beta). http://keplerebs.villanova.edu/. Accessed 16 Sep 2014

Kitze M, Neuhäuser R, Hambaryan V, Ginski C (2014) Superflares on the slowly rotating solar-type stars KIC10524994 and KIC07133671? Mon Notices R Astronomical Soc 442:3769-3776. doi:10.1093/mnras/stu1114

Landini M, Monsignori Fossi BC, Pallavicini R, Piro L (1986) EXOSAT detection of an X-ray flare from the solar type star Pi-prime UMa. Astronomy Astrophysics 157:217-222

Loomis E (1859) The great auroral exhibition of August 28th to September 4th. Am J Sci Arts, Second Series 32:318-335

Maehara H, Shibayama T, Notsu S, Notsu Y, Nagao T, Kusaba S, Honda S, Nogami D, Shibata K (2012) Superflares on solar-type stars. Nature 485:478-481. doi:10.1038/nature11063

Mitra-Kraev U, Harra LK, Williams DR, Kraev E (2005) The first observed stellar $X$-ray flare oscillation: constraints on the flare loop length and the magnetic field. Astronomy Astrophysics 436:1041-1047. doi:10.1051/0004-6361:20052834

Miyake F, Nagaya K, Masuda K, Nakamura T (2012) A signature of cosmic-ray increase in AD 774-775 from tree rings in Japan. Nature 486:240-242. 10.1038/nature 11123

Miyake F, Masuda K, Nakamura T (2013) Another rapid event in the carbon-14 content of tree rings. Nat Commun 4:1748. doi:10.1038/ncomms2783

Nakariakov VM, Melnikov VF (2009) Quasi-periodic pulsations in solar flares. Space Sci Rev 149:119-151. doi:10.1007/s11214-009-9536-3

Nogami D, Notsu Y, Honda S, Maehara H, Notsu S, Shibayama T, Shibata K (2014) Two sun-like superflare stars rotating as slow as the Sun. Publ Astronomical Soc Japan 66:L4. doi:10.1093/pasj/psu012

Notsu S, Honda S, Notsu Y, Nagao T, Shibayama T, Maehara H, Nogami D, Shibata K (2013a) High-dispersion spectroscopy of the superflare star KIC 6934317. Publ Astronomical Soc Japan 65:112. doi:10.1093/pasj/65.5.112

Notsu Y, Shibayama T, Maehara H, Notsu S, Nagao T, Honda S, Ishii TT, Nogami D, Shibata K (2013b) Superflares on solar-type stars observed with Kepler II. Photometric variability of superflare-generating stars: a signature of stellar rotation and starspots. Astrophysical J 771:127. doi:10.1088/0004-637X/771/2/127

Notsu Y, Honda S, Maehara H, Notsu S, Shibayama T, Nogami D, Shibata K (2015a) High dispersion spectroscopy of solar-type superflare stars. I. Temperature, surface gravity, metallicity, and $v \sin i$. doi:10.1093/pasj/psv001
Notsu Y, Honda S, Maehara H, Notsu S, Shibayama T, Nogami D, Shibata K (2015b) High dispersion spectroscopy of solar-type superflare stars. II. Stellar rotation, starspots, and chromospheric activities. doi:10.1093/pasj/psv002

Pevtsov AA, Fisher GH, Acton LW, Longcope DW, Johns-Krull CM, Kankelborg CC, Metcalf TR (2003) The relationship between X-ray radiance and magnetic flux. Astrophysical J 598:1387-1391. doi:10.1086/378944

Prša A, Batalha N, Slawson RW, Doyle LR, Welsh WF, Orosz JA, Seager S, Rucker M, Mjaseth K, Engle SG, Conroy K, Jenkins J, Caldwell D, Koch D, Borucki W (2011) Kepler eclipsing binary stars. I. Catalog and principal characterization of 1879 eclipsing binaries in the first data release. Astronomical J 141:83. doi:10.1088/0004-6256/141/3/83

Sammis I, Tang F, Zirin H (2000) The dependence of large flare occurrence on the magnetic structure of sunspots. Astrophysical J 540:583-587. doi:10.1086/309303

Schaefer BE (1989) Flashes from normal stars. Astrophysical J 337:927-933. doi:10.1086/167162

Schaefer BE, King JR, Deliyannis CP (2000) Superflares on ordinary solar-type stars. Astrophysical J 529:1026-1030. doi:10.1086/308325

Schrijver CJ, Beer J, Baltensperger U, G'udel M, Hudson HS, McCracken KG, Osten RA, Peter T, Soderblom DR, Usoskin IG, Wolff EW (2012) Estimating the frequency of extremely energetic solar events, based on solar, stellar, lunar, and terrestrial records. J Geophys Res (Space Physics) 117:8103. doi:10.1029/2012JA017706

Shibata K, Magara T (2011) Solar flares: magnetohydrodynamic processes. Living Rev Solar Phys 8:6. doi:10.12942/Irsp-2011-6

Shibata K, Isobe H, Hillier A, Choudhuri AR, Maehara H, Ishii TT, Shibayama T, Notsu S, Notsu Y, Nagao T, Honda S, Nogami D (2013) Can superflares occur on our Sun?. Publ Astronomical Soc Japan 65:49. doi:10.1093/pasj/65.3.49

Shibayama T, Maehara H, Notsu S, Notsu NagaoT, Honda S, Ishii TT, Nogami D, Shibata K (2013) Superflares on solar-type stars observed with Kepler. I. Statistical properties of superflares. Astrophysical J Suppl 209:5. doi:10.1088/0067-0049/209/1/5

Shimizu T (1995) Energetics and occurrence rate of active-region transient brightenings and implications for the heating of the active-region corona. Publ Astronomical Soc Japan 47:251-263

Slawson RW, Prša A, Welsh WF, Orosz JA, Rucker M, Batalha N, Doyle LR, Engle SG, Conroy K, Coughlin J, Gregg TA, Fetherolf T, Short DR, Windmiller G, Fabrycky DC, Howell SB, Jenkins JM, Uddin K, Mullally F, Seader S, Thompson SE, Sanderfer DT, Borucki W, Koch D (2011) Kepler eclipsing binary stars. II. 2165 eclipsing binaries in the second data release. Astronomical J 142:160. doi:10.1088/0004-6256/142/5/160

Smith JC, Stumpe MC, Van Cleve JE, Jenkins JM, Barclay TS, Fanelli MN, Girouard FR, Kolodziejczak JJ, McCauliff SeanD, Morris RL, Twicken JD (2012) Kepler presearch data conditioning II - a Bayesian approach to systematic error correction. Publ Astronomical Soc Pac 124:1000-1014. doi:10.1086/667697

Strassmeier KG (2009) Starspots. Astronomy Astrophysics Rev 17:251-308. doi:10.1007/s00159-009-0020-6

Stumpe MC, Smith JC, Van Cleve JE, Twicken JD, Barclay TS, Fanelli MN, Girouard FR, Jenkins JM, Kolodziejczak JJ, McCauliff SD, Morris RL (2012) Kepler presearch data conditioning I - architecture and algorithms for error correction in Kepler light curves. Publ Astronomical Soc Pac 124:985-999. doi:10.1086/667698

Tsurutani BT, Gonzalez WD, Lakhina GS, Alex S (2003) The extreme magnetic storm of 1-2 September 1859. J Geophys Res (Space Physics) 108:1268. doi:10.1029/2002JA009504

Veronig A, Temmer M, HansImeier A, Otruba W, Messerotti M (2002) Tempora aspects and frequency distributions of solar soft X-ray flares. Astronomy Astrophysics 382:1070-1080. doi:10.1051/0004-6361:20011694

Welsh BY, Wheatley J, Browne SE, Siegmund OHW, Doyle JG, O'Shea E, Antonova A, Forster K, Seibert M, Morrissey P, Taroyan Y (2006) GALEX high time-resolution ultraviolet observations of dMe flare events. Astronomy Astrophysics 458:921-930. doi:10.1051/0004-6361:20065304

Wichmann R, Fuhrmeister B, Wolter U, Nagel E (2014) Kepler super-flare stars: what are they? Astronomy Astrophysics 567:AA36. doi:10.1051/0004-6361/201423717

Wu C-J, Ip W-H, Huang L-C (2015) A study of variability in the frequency distributions of the superflares of G-type stars observed by the Kepler mission. Astrophysical J 798:92. doi:10.1088/0004-637X/798/2/92 